\title{
O papel do enfermeiro na oxigenoterapia: revisão narrativa da literatura
}

\section{The role of the hospital orderly in oxygenotherapy: narrative literature review}

Julya Carolline Folle Alves ${ }^{1}$, Adriana Fank², Laurindo Pereira de Souza ${ }^{3}$ (orcid.org/0000-0002-9890-2621), Marcia Guerino de Lima ${ }^{3}$

1. Discente do curso de Enfermagem da Faculdade de Ciências Biomédicas de Cacoal (FACIMED), Cacoal, RO, Brasil. 2. Pós-graduando em Enfermagem em Terapia intensiva Adulto e Pediátrico pela Faculdade de Ciências Biomédicas de Cacoal (FACIMED), Cacoal, R0, Brasil. 3. Docente do Departamento de Enfermagem da Faculdade de Ciências Biomédicas de Cacoal (FACIMED), Cacoal, RO, Brasil.

\section{Resumo}

Introdução: A oxigenoterapia é a administração de $\mathrm{O}^{2}$, em concentrações maiores que as existentes no ar ambiente. Objetivo: a proposta deste estudo de revisão narrativa da literatura foi conhecer a produção científica disponível acerca da importância do papel do enfermeiro e de suas ações quanto à oxigenoterapia. Método: trata-se de uma pesquisa de revisão narrativa da literatura, por meio de busca nas bases de dados virtuais e artigos de fontes sistematizadas que contemplavam o tema do estudo, utilizando os descritores: oxigenoterapia, enfermagem, conhecimento, sendo selecionado um total de 31 artigos, publicados entre os anos de 2004 a 2015, além da busca em livros que contemplavam a temática disponível na biblioteca Maria da Penha Lenci Campos da Faculdade de Ciências de Biomédicas de Cacoal/FACIMED. Resultados: os resultados foram sistematizados e analisados de modo a contribuir para o fortalecimento e conhecimento do enfermeiro a respeito dos cuidados com a oxigenoterapia e sua aplicabilidade. Conclusão: evidenciouse a necessidade de realização de novas pesquisas que abordem o papel do enfermeiro e os cuidados de enfermagem a respeito dos métodos não invasivos de oxigênio.

Palavras-chave: Oxigenoterapia. Enfermagem. Conhecimento.

\begin{abstract}
Introduction: Oxygen therapy is the administration of $\mathrm{O}^{2}$, in concentrations higher than those in ambient air. Objective: the purpose of this study of literature review was to know the available scientific production about the importance of the role of nurses and their actions regarding oxygen therapy. Method: this is a research of narrative review of the literature, carried out through search in the virtual databases and articles of systematized sources that contemplated the theme of the study, using the descriptors: oxygen therapy, nursing and knowledge. A total of 31 articles, published between the years of 2004 and 2015, were selected, as well as the search for books that were available in the Maria da Penha Lenci Campos library of the Faculty of Biomedical Sciences of Cacoal / FACIMED. Results: the results were systematized and analyzed so that it can contribute to the strengthening of nurses' knowledge regarding oxygen therapy care and its applicability. Conclusion: it was evidenced the necessity of conducting new research works that address the role of nurses and nursing care regarding non-invasive methods of oxygen.
\end{abstract}

Keywords: Oxygen therapy. Nursing. Knowledge.

\section{INTRODUÇÃO}

A respiração tem como objetivo prover oxigênio $\left(\mathrm{O}_{2}\right)$ aos tecidos e remover o dióxido de carbono ${ }^{1}$.

Segundo Guyton \& Hall ${ }^{1}$, no processo de inspiração ocorrem todas as contrações dos músculos respiratórios; já na expiração, o processo é passivo, pois é causado pelo recuo elástico dos pulmões e da caixa torácica. Reforça ${ }^{1}$, ainda que o processo de inspiração pode ser dividido em três frações: aquela necessária para expandir os pulmões contra as forças elásticas do pulmão e do tórax, chamada de trabalho de complacência ou trabalho elástico; aquela necessária para sobrepujar a viscosidade pulmonar e das estruturas da parede torácica, chamada de trabalho de resistência tecidual e aquela necessária para sobrepujar a resistência aérea ao movimento de ar para dentro dos pulmões, chamada de trabalho de resistência das vias aéreas.

Os distúrbios respiratórios devem ser diagnosticados e tratados de maneira adequada a fim de evitar complicações. Isso exige que os profissionais de enfermagem tenham melhor preparo técnico para reconhecer, encaminhar e acompanhar os pacientes ${ }^{2}$.

A administração de oxigênio é uma das mais importantes modalidades de tratamento de hipoxemia, ocasionada por insuficiência respiratória ${ }^{3}$. Sendo as principais indicações de oxigenoterapia, pacientes com pressão arterial de oxigênio $\left(\mathrm{PaO}_{2}\right)<60 \mathrm{mmHg}$ ou saturação periférica de oxigênio $\left(\mathrm{SpO}_{2}\right)$ $<90 \%$, em ar ambiente, $\mathrm{SpO}_{2}<88 \%$ durante deambulação, exercício ou sono em portadores de doenças cardiorrespiratórias, infarto agudo do miocárdio (IAM), intoxicação por gases, e envenenamento por cianeto ${ }^{28}$.

A oxigenoterapia é a administração de $\mathrm{O}_{2}$, em concentrações maiores que a existente no ar ambiente. Pode ser realizada através de cateter nasal tipo óculos que possibilita uma FiO2 
entre $24 \%$ e $40 \%$, máscara facial ou de Venturi que administra níveis de $\mathrm{FiO}_{2}$ constante e pré-estabelecidos, com uma $\mathrm{FiO}_{2}$ de até $60 \%$, as máscaras com reservatório alcançam uma $\mathrm{FiO}_{2}$ de 60 a $80 \%$ a 10 litros por minuto. As máscaras sem reinalação apresentam válvulas unidirecionais que evitam a reinalação e podem alcançar $\mathrm{FiO}_{2}$ de 80 a $95 \%$ e outros dispositivos invasivos ${ }^{4}$. A oxigenoterapia tem por objetivo corrigir ou minimizar as deficiências de oxigênio, melhorando a troca gasosa e reduzindo o esforço respiratório e o estresse do miocárdio 5 .

Quando o oxigênio é administrado de forma desnecessária, em altas ou baixas concentrações, pode acarretar estreitamento dos vasos de forma sistêmica e aumento da pressão arterial sistêmica, resultando na diminuição do débito cardíaco (DC) sendo nocivo ao paciente ${ }^{6}$. Nos neonatos, concentrações exacerbadas de oxigênio podem ocasionar complicações como a displasia broncopulmonar e a retinopatia da prematuridade ${ }^{7}$.

É de fundamental importância que o enfermeiro tenha conhecimento sobre os dispositivos disponíveis, as razões de sua eleição, as vantagens e desvantagens de cada método adotado e os cuidados relacionados, bem como o fluxo de oxigênio adequado e a fração fornecida de oxigênio inspirado $\left(\mathrm{FiO}_{2}\right)^{5}$.

A oxigenoterapia é indicada em pacientes que apresentam insuficiência respiratória aguda ou saturação de oxigênio inferior a 94\%. A técnica corrige a hipoxemia e consequentemente reduz o trabalho respiratório e cardíaco ${ }^{6,9}$.

É um tratamento não invasivo de grande importância clínica; por ser considerada uma medicação de prescrição médica, cabe ao enfermeiro conhecer a dosagem adequada, a forma de administração e a pressão necessária; além disso, também é papel do enfermeiro avaliar o paciente por meio da anamnese, do exame físico e também por meio do monitoramento de saturação de $\mathrm{O}_{2}$ do paciente. Diante disso exigem-se, profissionais especializados, aptos para diagnosticar e intervir com brevidade e competência nas intercorrências.

A percepção dos autores sobre a temática envolvendo a enfermagem detectou escassez de publicações. Assim, entende-se a relevância dessa pesquisa com o intuito de estabelecer o suporte e a consolidação dos conhecimentos necessários aos enfermeiros, para o desenvolvimento da boa prática profissional, concernentes à função da oxigenoterapia.

Desse modo, o objetivo deste estudo foi conhecer a produção científica disponível acerca da importância do papel do enfermeiro e de suas ações quanto à oxigenoterapia, o que justifica a necessidade deste estudo para ampliar o campo de conhecimento do enfermeiro, visando a uma atuação segura junto ao paciente, com o propósito de garantir uma assistência de qualidade.

\section{MÉTODOS}

Trata-se de uma revisão narrativa da literatura realizada por meio de uma revisão bibliográfica, que tem por finalidade apontar fundamentações científicas acerca da importância do enfermeiro e de suas ações no que diz respeito à oxigenoterapia, a fim de estabelecer suas evidências e consolidar os conhecimentos necessários para a boa prática profissional.

Segundo Rother $^{8}$, revisões narrativas são estudos compostos por análises da literatura publicadas em artigos de revistas impressas ou eletrônicas, livros na interpretação e apreciação crítica do autor; são produções pertinentes para descrever e discutir a situação das produções científicas acerca de determinado assunto.

A busca das produções bibliográficas ocorreu no período compreendido entre março e maio de 2016, em periódicos disponíveis na internet publicados no período entre 2004 e 2015, nos idiomas português, inglês ou espanhol por meio da busca em base de dados: BDENF, LILACS, SciELO e artigos de fontes sistematizadas que contemplavam o tema do estudo; foram utilizados os descritores de Ciências da Saúde DeCS: "oxigenoterapia", "enfermagem" e "conhecimento"; utilizou-se o operador booleano AND e OR, para associação/combinação de palavras, restringindo, assim, a busca nos bancos de dados.

Foram definidos os seguintes critérios de inclusão: textos disponíveis online na íntegra, na forma de artigos originais, revisão bibliográfica, além de relatos de experiência e de casos que respondessem ao objetivo do estudo. Os critérios de exclusão foram artigos que não contemplavam o objetivo da pesquisa e que não compreendiam período de estudo.

Inicialmente, realizou-se a leitura do título e resumo dos artigos encontrados. Foram identificados 50 artigos, conforme a aplicação dos critérios de inclusão. Após a leitura detalhada dos textos, foram excluídas 19 publicações que não estavam diretamente relacionadas ao conteúdo da atual pesquisa. Assim, a amostra final foi composta por 31 artigos científicos, além da busca em livros que contemplavam temas sobre oxigenoterapia invasiva e não invasiva.

Após o processo de seleção, procedeu-se à leitura detalhada dos 31 artigos, analisando os conteúdos que contemplavam o objetivo da pesquisa, sendo os resultados e discussões sistematizados e categorizados por similaridade do conteúdo em três categorias temáticas: conhecimento e habilidades necessárias concernentes à indicação e formas de administração de oxigênio/ ações da enfermagem diante do paciente em oxigenoterapia por ventilação invasiva e não invasiva e oxigenoterapia e suas implicações para a enfermagem.

Considerando-se os aspectos éticos, nesta revisão bibliográfica é assegurada a autoria dos artigos pesquisados, de forma que todas as fontes bibliográficas e artigos científicos foram citados. Neste estudo, é dispensável o parecer do Comitê de Ética em Pesquisa, pois ele não envolveu seres humanos e instituições como pesquisa.

\section{RESULTADOS E DISCUSSÃO}

Segundo Barros $^{9}$, as alterações no padrão ou na frequência respiratória do paciente pode ser um dos indicadores da necessidade de oxigenoterapia. Modificações no estado mental, dispneia, variação da pressão arterial, taquicardia e 
extremidades frias, são sinais clínicos que indicam hipoxemia.

Pesquisadores como Porth \& Matfin revelam ${ }^{10}$ que a hipoxemia consiste em uma redução nos níveis de pressão parcial de oxigênio $\left(\mathrm{PaO}_{2}\right)$. Ela pode ser ocasionada pela baixa quantidade de oxigênio (O2) no ar, alterações respiratórias ou disfunções neurológicas. Por isso, a hipoxemia é mais bem definida como a diminuição da $\mathrm{PaO}_{2}$ abaixo dos níveis fisiológicos.

Porth \& Matfin reforçam ${ }^{10}$ ainda que os mecanismos pelos quais as doenças respiratórias levam a uma significativa redução na $\mathrm{PaO}_{2}$ são: hipoventilação, disfunções nas trocas gasosas, circulação inadequada de sangue nos alvéolos e má combinação entre ventilação e perfusão. Para a detecção da hipoxemia, podem ser utilizados métodos invasivos e não invasivos, que demonstram os valores de $\mathrm{PaO}_{2}$ e de saturação de oxigênio $\left(\mathrm{SaO}_{2}\right)$; são eles a gasometria e a oximetria de pulso.

Para Mota \& Queiroz ${ }^{11}$ a gasometria é um exame invasivo que analisa os gases no sangue arterial; ela tem por objetivo revelar valores da pressão parcial de gás carbônico $\left(\mathrm{PcO}_{2}\right)$, oxigênio $\left(\mathrm{PaO}_{2}\right)$, e bicarbonato $\left(\mathrm{HCO}^{3}\right)$. Com isso, é possível avaliar a adequada ventilação, o equilíbrio ácido-base e a oxigenação ${ }^{12}$.

Medições não invasivas da saturação arterial de $\mathrm{O}_{2}$ da hemoglobina podem ser obtidas usando-se um instrumento chamado oxímetro de pulso. Embora ele não seja tão preciso quanto as medições de gases sanguíneos arteriais, ele fornece o meio para a monitoração não invasiva e contínua de $\mathrm{SaO}_{2}$, que é um indicador útil do estado da oxigenação sanguínea ${ }^{10}$.

As formas de administração de oxigênio podem ser realizadas por meio de dispositivos de baixo e alto fluxo, determinadas de acordo com a clínica apresentada pelo paciente e os resultados dos exames complementares.

De acordo com Birolini \& Atallah ${ }^{15}$, os dispositivos de baixo fluxo fornecem um fluxo constante de oxigênio e permitem a mistura do ar ambiente com o ar enriquecido de $\mathrm{O}_{2}$; eles são representados pela cânula nasal tipo óculos, cânula nasal pela máscara de alta concentração, máscara facial simples e máscara de reservatório de $\mathrm{O}_{2}$. Os dispositivos de alto fluxo são representados pela máscara de venturi que fornece fluxo constante de $\mathrm{O}_{2}$, entretanto com maior $\mathrm{FiO}_{2}$.

Barbas et al., ${ }^{16}$ revelam que a enfermagem como membro da equipe multi e interdisciplinar no âmbito nosocomial, contribui de maneira significativa para as atividades assistenciais e administrativas, que compreendem o suporte invasivo e não invasivo nos clientes que estão em uso de ventilação mecânica.

Para Carvalho ${ }^{17}$, a ventilação mecânica invasiva acontece por meio de aparelhos que distribuem ar para as vias aéreas. Ela faz uso de algum tipo de endoprótese e/ou tubo para oferecer suporte ventilatório, podendo ser orotraqueal, nasotraqueal ou traqueostomia para a realização do correto desempenho ${ }^{18}$. Contribuindo com os conceitos apresentados anteriormente, Melo et al.,19 reforçam que este tipo de suporte é necessário para pacientes que apresentem mudança do padrão respiratório que impossibilite que ocorra a hematose e o suprimento de oxigênio para as células.
A ventilação não invasiva (VNI) é a aplicação de um suporte ventilatório sem utilização de métodos invasivos da via aérea, sendo administrada por intermédio de máscaras nasais ou faciais $^{21,22}$. É importante ressaltar que, para a sua utilização, o paciente deve encontrar-se desperto e cooperativo, tenha estabilidade hemodinâmica e que os reflexos das vias aéreas superiores estejam preservados para evitar a broncoaspiração ${ }^{15}$.

O método de oferta de oxigenoterapia que oferece maior conforto é a máscara nasal; no entanto, a sensibilidade das narinas mediante a pressão do fluxo de ar e o escape de ar pela cavidade oral restringem sua utilização. A máscara oronasal ou facial é a interface que permite maior volume corrente comparado com a máscara nasal, pois possibilita uma correção mais rápida das trocas gasosas ${ }^{23}$.

A ventilação não invasiva melhora a oxigenação e o prognóstico clínico de clientes com diferentes tipos de patologias respiratórias agudas, diminui o risco de pneumonia associada à ventilação mecânica invasiva, à necessidade de intubação, além de minimizar o risco de óbito, mas o êxito de seu uso depende da colaboração do paciente ${ }^{24}$

A equipe de enfermagem, por participar e ser responsável de forma ativa pela prestação de cuidados, deve atentar-se continuamente, de forma segura, quanto ao manuseio, conservação e manutenção dos equipamentos disponíveis no setor, realizando troca de circuitos, filtros e umidificadores dentro dos prazos de validade preconizados conforme os protocolos da $\mathrm{CCIH}$ de cada instituição, além de prestar cuidados de forma rigorosa no momento do banho e da mudança de decúbito, pois o doente pode sofrer sérias instabilidades hemodinâmicas; reforça ainda que o cuidado com a cavidade oral, o cuidado com a nutrição seja pela boca ou por meio de dispositivos como sonda enteral ou outros meios de alimentação, é fundamental para o pacien$\mathrm{te}^{16}$.

De acordo com Barbas et al. ${ }^{25}$ pacientes em utilização de VM devem fazer uso de umidificação e aquecimento ativos, a fim de evitar a oclusão do tubo; também preconizam que a troca de filtros umidificadores "narizinhos' seja realizada a cada sete dias em posição perpendicular ao paciente, ou sempre que necessário.

E de suma importância, antes de realizar qualquer procedimento que possa interferir na hemodinâmica do paciente, fazer a avaliação dos sinais vitais, análise e registro dos parâmetros do ventilador mecânico e manter a monitorização cardíaca e da saturação de forma contínua, principalmente no momento do banho no leito e em outras mobilizações ${ }^{16}$, pois o paciente que faz uso de VM está em estado crítico e fazendo uso de diversos equipamentos; o desposicionamento de qualquer acessório pode oferecer risco à vida do paciente ${ }^{26}$.

Assim, a mudança de decúbito requer técnicas e cuidados especiais $^{26}$ recomenda-se que se utilize um traçado móvel e que seja realizada por, no mínimo, dois profissionais, pois há a necessidade de que um profissional esteja atento à estabilidade do dispositivo ventilatório ${ }^{16,27}$. Incumbe-se que o banho no leito deve ser realizado na técnica, e que mantenha a cabeça centralizada, para que se evite a ocorrência de extubação precoce e, 
em consequência, uma hipoxemia ${ }^{27,28}$.

A equipe de enfermagem é responsável pela administração medicamentosa, inclusive a de uso inalatório que é o caso da oxigenoterapia. Cabe ao enfermeiro a orientação aos funcionários quanto à realização adequada da técnica de administração, a educação direta e as orientações ao paciente; além disso, o enfermeiro, com sua prática, contribui consideravelmente para o tratamento de pacientes com enfermidades respiratórias por meio da realização da anamnese e do exame físico do tórax ${ }^{9,30}$.

O papel da enfermagem mediante a assistência ventilatória tem como objetivo manter a perfusão e a oxigenação adequada dos órgãos e tecidos a fim de prevenir a hipóxia, por meio da monitorização de seus sinais e sintomas ${ }^{31}$.

De acordo com o COREN/SP 014/2012 32 , o enfermeiro pode prescrever a instalação ou troca do cateter nasal, desde que se realize uma avaliação sistemática anterior do cliente, podendo, assim, ser prescrito em qualquer situação, uma vez respeitados os protocolos. Vale ressaltar que a presença de normas a respeito é fator de grande relevância para a padronização das ações da equipe de saúde.

Os cuidados de enfermagem relacionados ao fornecimento de oxigênio incluem a avaliação e inspeção das narinas, antes da aplicação da técnica e diariamente com o objetivo de conforto e prevenção, além de garantir uma melhor oferta de $\mathrm{O}_{2}$. $\mathrm{O}$ enfermeiro deve atentar-se quanto à fixação e à proteção da pele fazendo uso da umidificação do oxigênio ofertado com água destilada estéril, a monitorização da adaptação do paciente ao método por meio da avaliação dos sinais clínicos de hipóxia e da oximetria de pulso, a comunicação ao médico sobre as alterações apresentadas e a rotina de troca e processamento dos materiais ${ }^{5}$.

Os enfermeiros desempenham um papel fundamental na vigilância da pessoa e na prevenção de complicações que possam comprometer a saúde e retardar sua recuperação, fazendo-se importante a utilização de dispositivos novos ou mais eficientes para uma vigilância e uma monitorização eficaz ${ }^{13}$.

Segundo Barbosa ${ }^{33}$, a enfermagem precisa estabelecer um padrão para ter autonomia na prática profissional, caracterizando por meio dos julgamentos clínicos que irão dar origem aos diagnósticos de enfermagem, as prescrições e avaliações de enfermagem com o propósito de garantir uma assistência humana, segura e de qualidade ao cliente.

Cuidar do ser humano consiste em fazê-lo sentir-se melhor, mesmo em condições adversas. No ato do cuidar, surge a necessidade de um processo direcionado, constituído de ações e intervenções que promovam uma assistência diferenciada e sistematizada ${ }^{34}$.

A sistematização da assistência de enfermagem (SAE) é um método para coordenar a assistência fundamentada em princípios científicos. Com o intento de reconhecer as condições de saúde-doença e identificar a necessidade de assistência de enfermagem, além de contribuir para o sujeito, a família e a comunidade no âmbito da prevenção, promoção, recuperação e reabilitação da saúde ${ }^{35}$.

De acordo com NANDA international ${ }^{36}$ o processo de enfermagem integra a coleta de dados, diagnósticos de enfermagem, planejamento, estabelecimento de resultados, intervenção e avaliação.

O profissional enfermeiro (a) no rol da enfermagem é o único que pode prescrever os Diagnósticos de Enfermagem (DE) que são a segunda etapa do processo de enfermagem (PE), possibilitando a identificação dos problemas, e assim restabelecendo o estado clínico do cliente e a promoção da saúde ${ }^{37}$.

As prescrições de enfermagem remetem aos cuidados que devem ser realizados, e é fundamentado por meio do pensamento crítico e das manifestações clínicas, analisadas pelo profissional enfermeiro fundamentado em evidências científicas para a meIhoria do cliente e em resultado a um $\mathrm{DE}^{38}$.

Corroborando Tanurre \& Pinheiro ${ }^{37}$ que afirmam que esse processo é um desafio, pois exige que o enfermeiro tenha conhecimento técnico-científico e pensamento crítico ao analisar os dados coletados na anamnese e no exame físico para que possa assumir por intermédio do cuidado o que está propondo na prescrição de enfermagem.

Para a definição dos diagnósticos de enfermagem, é necessário valer-se de suas características definidoras e fatores relacionados segundo o que é abordado pela NANDA International ${ }^{36}$. Os fatores evidenciados ou caracterizados são parâmetros clínicos passíveis de observação que se agrupam e sugerem um diagnóstico, ou seja, o agente etiológico. Os fatores relacionados são fatores ou situações que integram todos os diagnósticos de enfermagem com foco no problema, e o que se considera como manifestações clínicas ${ }^{36}$.

\section{CONCLUSÕES}

O profissional da enfermagem deve atuar de forma completa no cuidado ao paciente portador de enfermidade respiratória e em uso de oxigenoterapia, por meio do processo científico de sistematização de assistência de enfermagem, prestando uma assistência sistemática e holística pautada em cuidados com embasamentos técnicos e científicos. Para isso, é de fundamental importância que o enfermeiro disponha de conhecimento sobre fisiologia pulmonar, assim como responsabilidade mediante suas competências profissionais neste âmbito, evitando complicações e contribuindo para a melhora do estado clínico do paciente.

Frente ao exposto, destaca-se a necessidade de aquisição de conhecimentos pela equipe de enfermagem, sendo de suma importância desenvolver capacitação e educação permanente, além da implantação de protocolos baseado em evidências científicas, que são fundamentais para padronizar e uniformizar os conhecimentos e subsidiar os profissionais na prestação de um cuidado seguro.

Encontraram-se limitações na busca por artigos relacionados 
à assistência de enfermagem a pacientes em oxigenoterapia, mas este artigo vem contribuir para a atuação do enfermeiro de maneira segura, por meio da sistematização da assistência de Enfermagem, prescrevendo os cuidados que deverão levar ao cliente uma assistência com qualidade.
Destarte, este estudo reforça a importância de novas pesquisas sobre esta temática, no sentido de contribuir com as ações da enfermagem para as práticas baseadas em evidências (PBE).

\section{REFERÊNCIAS}

1 Guyton AC, Hall JE. Tratado de fisiologia médica. 12 ed. Rio de Janeiro: Elsevier; 2011.

2. Souza ABG, Chaves LD, Silva MCM. Enfermagem em clínica médica e cirúrgica: teoria e prática. São Paulo: Martinari; 2014. p. 519. Volume 1.

3. Zeferino MT, Silva AS. Diretrizes para oxigenoterapia domiciliar. São Paulo: Segmento Farma Editores; 2004.

4. Donoso MTV, Silqueira SMDF, Barbosa RDCGDA, Vasconcelos TRDC, Anastácio VLA. Oxigenoterapia e ventilação mecânica em atenção domiciliar. Belo Horizonte: Nescon UFMG; 2013.

5. Nepomuceno RM, Silva LD, Silva DB, Sergio FR, Alexandre PS, Silva FZ. Revisão bibliográfica acerca das recomendações para a oxigenoterapia do paciente crítico com métodos não invasivos. Nursing (São Paulo). 2012;15(172):487-92.

6. Piegas LS, Timerman A, Feitosa GS, Nicolau JC, Mattos LAP, Andrade MD, et a . V Diretriz da Sociedade Brasileira de Cardiologia sobre Tratamento do Infarto Agudo do Miocárdio com Supradesnível do Segmento ST. Arq Bras Cardiol. 2015 Ago;105(2 Suppl 1 ):1-121.

7. Pereira SA. O uso do oxigênio em prematuros: "o que os olhos não veem e o pulmão sente". Movimenta. 2012; 5(3):207-8.

8. Rother ET. Revisão sistemática X revisão narrativa. Acta paul enferm. 2007 AbrJun; 20(2): v-vi. doi: http://dx.doi.org/10.1590/S0103-21002007000200001.

9. Barros ALBL, Andriolo A, Isabella APJ, Sá AC, Bettencourt ARC, Leite AL et al. Anamnese e exame físico: avaliação diagnóstica de enfermagem no adulto. 2 ed., Porto Alegre: Artmed; 2010.

10. Porth CM, Matfin G. Fisiopatologia. 8 ed. Rio de Janeiro: Guanabara Koogan; 2010; p. 717. Volume 1.

11. Mota IL, Queiroz RS. Distúrbios do equilíbrio ácido básico e gasometria arterial: uma revisão crítica. Rev Digital. 2010;14(141).

12. Sanderson LGD. Gasometria arterial: artigo de revisão interpretação. [monografia]. Porto Ferreira: 2012.

13. Carreira A. Capnografia em pessoa submetida a ventilação mecânica não invasiva [dissertação]. Setúbal (PT): Instituto Politécnico de Setúbal; 2015.

14. Kock KDS, Rocha PAC, Silvestre JCDC, Coelho D, Leite KR. Adequações dos dispositivos de oxigenoterapia em enfermaria hospitalar avaliadas por oximetria de pulso e gasometria arterial. ASSOBRAFIR Ciência. 2014 Abr; 5(1):53-64.

15. Birolini D, Atallah NA, Àlvaro Nagib. Atualização de terapêutica de Prado, Ramos e Valle: urgências e emêrgencias. São Paulo: Artes médicas; 2012.

16. Barbas CSV, Ísola AM, Farias AMC, Cavalcanti AB, Gama AMC, Duarte ACM, et al. Recomendações brasileiras de ventilação mecânica 2013. Parte 2. Rev Bras Ter Intensiva. 2014; 26(3):215-39.

17. Carvalho CRR, Toufen C Junior, Franca SA. Ventilação mecânica: princípios, análise gráfica e modalidades ventilatórias. J bras pneumol. 2007; 33(suppl 2): 54-70. doi: http://dx.doi.org/10.1590/S1806-37132007000800002.

18. Vargas JS, Rezende MS. Comunicação: equipe de enfermagem e paciente em ventilação mecânica. Rev Enferm UFSM. 2011;1(3):412-19.
19. Melo EM, Teixeira CS, Oliveira RTD, Almeida DTD, Veras JEGLDF, Frota NM, et al. Nursing care of hospitalised patients receiving mechanical ventilation in intensive care units. Rev Enf Ref. 2014 Mar;4(1):55-63. doi: http://dx.doi. org/10.12707/RIII1316.

20. Marcó R. Avaliação da ventilação mecânica não invasiva após a ventilação mecânica convencional [dissertação]. São Paulo (SP): Faculdade de Ciências Médicas da Santa Casa de São Paulo; 2010.

21. Ferreira S, Nogueira C, Conde S, Taveira N. Ventilação não invasiva. Rev Port Pneumol. 2009;15(4):655-67.

22. Sarmento GJV. Princípios e práticas de ventilação mecânica. São Paulo: Manole; 2009.

23. Schettino GPP, Reis MAS, Galas F, Park M, Franca S, Okamoto V. Ventilação mecânica não invasiva com pressão positiva. J Bras Pneumol. 2007;33(suppl.2):92-105. doi: http://dx.doi.org/10.1590/S180637132007000800004 .

24. Rahal L, Garrido AG, Cruz JRJ. Ventilação não invasiva: quando utilizar? Rev Assoc Med Bras. 2005; 51(5):245-6. doi: http://dx.doi.org/10.1590/S010442302005000500007.

25. Barbas CSV, Ísola AM, Farias AMC, Cavalcanti AB, Gama AMC, Duarte ACM, et al . Recomendações brasileiras de ventilação mecânica 2013. Parte I. Rev Bras Ter Intensiva. 2014;26(2):89-121. doi: http://dx.doi.org/10.5935/0103507X.20140017.

26. Castellões TMFW, Silva LD. Ações de enfermagem para a prevenção da extubação acidental. Rev Bras Enferm. 2009; 62(4):540-5. doi: http://dx.doi. org/10.1590/S0034-71672009000400008.

27. Moons P, Sels K, De Becker W, De Geest S, Ferdinande P. Development of a risk assessment tool for deliberate selfextubation in intensive care patients. Inten Care Med. 2004; 30(7):1348-55. doi: http://dx.doi.org/10.1007/s00134004-2228-2.

28. Antonucci LAO, Savino MJP. Paciente com ventilação espontânea prejudicada: uma revisão integrativa das intervenções de enfermagem no uso da respiração artificial. Rev Saúde Com. 2014;10(1): 96-108. doi: http://dx.doi. org/10.22481/rsc.v10i1.216.

29. Silva SG, Nascimento ERP, Salles Raquel K. Pneumonia associada à ventilação mecânica: discursos de profissionais acerca da prevenção. Esc Anna Nery. 2014 Abr-Jun;18(2):290-5. doi: http://dx.doi.org/10.5935/1414-8145.20140042.

30. Maciel PP. Efeito da intervenção educativa sobre a técnica de administração de medicamentos inalatórios por profissionais de enfermagem [monografia]. Porto Alegre (RS): Universidade Federal do Rio Grande do Sul; 2009.

31. Nepomuceno RM. Condutas de enfermagem diante a ocorrência de alarmes ventilatórios em pacientes críticos [dissertação]. Rio de Janeiro (RJ): Universidade do Estado do Rio de Janeiro; 2007.

32. São Paulo. Conselho Regional de Enfermagem. Parecer Coren-SP 014/2012, de 10 de agosto de 2012. Administração de oxigênio medicinal, sem prescrição médica, por meio de cateter nasal em situação de emergência. São Paulo: COREN; 2012.

33. Barbosa PMK, Guimarães AA, Santos VC, dos Anjos KF. "Troca de gases 
prejudicada": intervenções de enfermagem. Rev Eletr Fainor. 2009 Jan-Dez; 2(1):33-45.

34. Marques SM, Brito KCG, Fernandes CM, Vieira AG. Sistematização da assistência de enfermagem na UTI: perspectivas dos enfermeiros da cidade de Governador Valadares. REME Rev Min Enferm. 2008 Out-Dez;12(4):469-76. doi http://www.dx.doi.org/S1415-27622008000400004.

35. Truppel TC, Meier MJ, Calixto RC, Peruzzo SA, Crozeta K. Sistematização da Assistência de Enfermagem em Unidade de Terapia Intensiva. Rev Bras Enferm. 2009 Mar-Abr; 62(2): 221-7. doi: http://dx.doi.org/10.1590/S003471672009000200008
36. North American Nursing Diagnosis Association (NANDA). Diagnósticos de Enfermagem da Nanda: definições e classificação: 2015-2017. Porto Alegre: Artmed; 2015

37. Tanurre MC, Pinheiro AM. Sistematização da assistência de enfermagem. 2. ed. Rio de Janeiro: Guanabara Koogan; 2010.

38. Santos VFR, Figueiredo AEPL. Intervenção e atividades propostas para o diagnóstico de enfermagem-ventilação espontânea prejudicada. Acta paul enferm. 2010; 23(6):824-30. doi: http://dx.doi.org/10.1590/S0103 21002010000600017.

\section{Como citar este artigo/How to cite this article:}

Alves JCF, Fank A, Souza LP, Lima MG. O papel do enfermeiro na oxigenoterapia: revisão narrativa da literatura. J Health Biol Sci. 2018 Abr-Jun; 6(2):176-181. 